\title{
Automatic Brachial Ankle Pulse Wave Velocity Measurements for Vascular Damage Assessments
}

\author{
R Gonzalez ${ }^{1,2}$, O Morales ${ }^{2}, \mathrm{~J} \mathrm{Delgado}^{2}$, JM Padilla ${ }^{2}$, \\ JM Ferrero ${ }^{1}$, J Saiz $^{1}$ \\ ${ }^{1}$ Universidad Politecnica de Valencia, Spain \\ ${ }^{2}$ Instituto Tecnologico de Morelia, Mexico
}

\begin{abstract}
An automatic brachial ankle pulse wave velocity device was developed using plethysmographic method based on blood pressure cuffs wrapped on arm brachial artery and tibial artery of ankle. Three electrodes of electrocardiogram were placed on ventral surface of both wrists and medial side of right ankle. The acquired signals of volume pulse and ECG were displayed. The pulse transit time between brachium and ankle was calculated as the time difference between maximal time derivative points and foot-to-foot pulse waves originated by the same QRS complex. A study was carried out with 39 people, 22 healthy volunteers and 17 patients with diagnosed cardiovascular diseases. A t-tested distributions of brachial ankle pulse wave velocity were performed. They showed that using maximal time derivative is more accurate than foot-to-foot pulse wave both in patients with cardiovacular disease and in healthy volunteers.
\end{abstract}

\section{Introduction}

During each heart beat a pulse wave travels from the heart down the arterial wall in advance of blood flow. The more rigid the wall of the artery, the faster the wave moves. When the wave hits the major branching points, such as at the renal and femoral arteries, these waves are reflected back so that they reverse direction and travel back to their point of origin [1,2].

Normally, the reflected wave gets back to the starting point after the aortic valve is closed. This amplifies diastolic pressure and facilitates blood flow to the coronary arteries. However, the increased wave velocity of the initial wave and the subsequent reflected wave that occurs with age means the wave can get back to the point of origin before the aortic valve closes which increases systolic rather than diastolic pressure. It also decreases the contribution of the reflected wave to the filling of the coronary arteries. These changes can have significant clinical implications in terms of coronary artery blood flow and can contribute to an increase in systolic blood pressure.

The arterial pulse is a viscous-fluid shock wave that is initiated by blood ejected from the heart as is shown in figure 1 . This wave travels away from the heart at a speed termed the pulse wave velocity (PWV). The PWV increases during the course of a number of diseases, and this increase is often attributed to arterial stiffness [3]. As the pulse wave approaches a point in an artery, the pressure rises as does the pressure gradient. This pressure gradient increases the rate of blood flow ahead of the wave. The rate of blood flow ahead of the wave decreases with distance because the pressure gradient also decreases with distance ahead of the wave. Consequently, the amount of blood per unit length in a segment of an artery increases ahead of the wave, and this increase stretches the wall of the artery. As a result, the tension in the wall increases, and this results in an increase in the pressure of blood in the artery. In elastic vessels, the reflected wave tends to arrive back at the aortic root during diastole, serving to augment diastolic pressure and hence improve coronary artery perfusion.

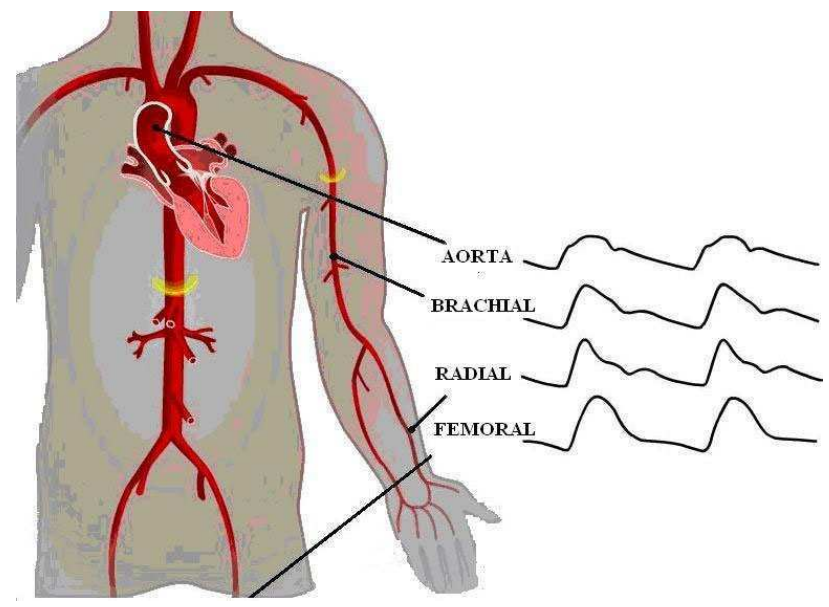

Figure 1. Arterial pulse wave propagation 
The condition of the major arteries is the key to better prevention and diagnosis of cardiovascular related illness. Arterial stiffness is a major cause of cardiovascular disease. Increases in arterial stiffness increase central systolic and pulse pressure, and demand on the left ventricle, and decrease the perfusion pressure through the coronary arteries, thereby increasing the risk of heart attacks, heart failure and stroke.

Pulse wave velocity is a well established technique for obtaining a measure of arterial stiffness between two locations in the arterial tree [4]. The velocity of the pulse wave along an artery is dependent on the stiffness of that artery - the stiffer of the artery walls, the higher the velocity. Pulse wave velocity provides information on the distensibility of the local vessel being studied, rather than on systemic arterial stiffness; distensibility being inversely related to stiffness.

The pulse wave velocity is the speed at which the forward pressure wave is transmitted from the aorta through the vascular tree. It is calculated by measuring the time taken for the arterial waveform to pass between two points a measured distance apart, and involves taking readings from the two sites simultaneously, or gating separate recordings to a fixed point in the cardiac cycle, usually the R wave of the ECG [5]. PWV describes how quickly a blood pressure pulse travels from one point to another in the human body. See figure 2. The time difference between these two locations is known as the pulse transit time $(\Delta \mathrm{t})$. Thus, $\mathrm{PWV}=$ Distance/ $\Delta \mathrm{t}$.

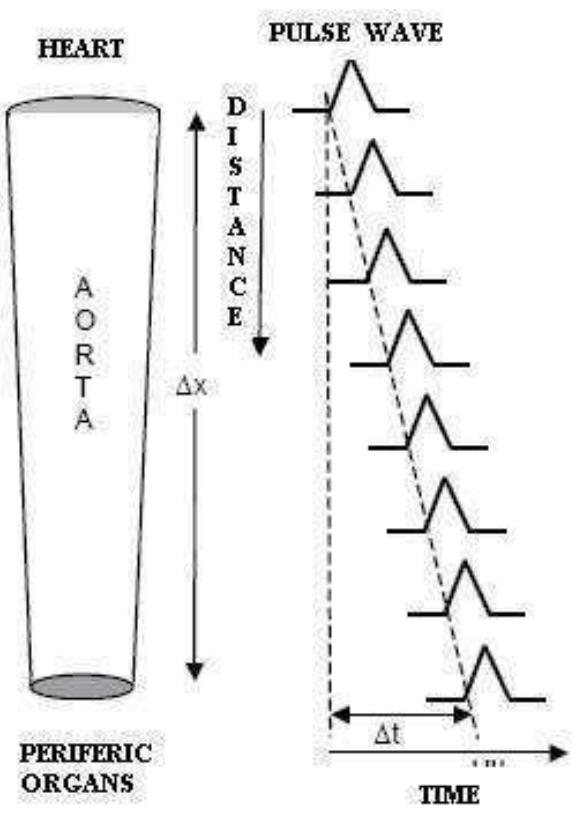

Figure 2. Pulse wave propagation.
The important point is the reference point on the waveforms. Usually, the pulse transit time of pulse wave velocity is determined by the foot-to-foot pulse wave as it avoids the confounding influence of wave reflection [6]. In people with diagnosed cardiovascular diseases, the shape of waveform could be irregular, making the automatic pulse wave velocity measurements non consistent.

The aim of this work is to increase the accuracy of automatic brachial ankle pulse wave velocity measurements by using the maximal time derivative point of waveform rather than foot-to-foot pulse wave as pulse transit time.

\section{Methods}

An automatic brachial ankle pulse wave velocity device (baPWV) was developed using plethysmographic method based on blood pressure cuffs wrapped on arm brachial artery and tibial artery of ankle [7]. Three electrodes of electrocardiogram were placed on ventral surface of both wrists and medial side of right ankle. See figure 3. The pulse transit time between brachium and ankle was calculated as the time difference between maximal time derivative points and foot-to-foot pulse waves originated by the same QRS complex.

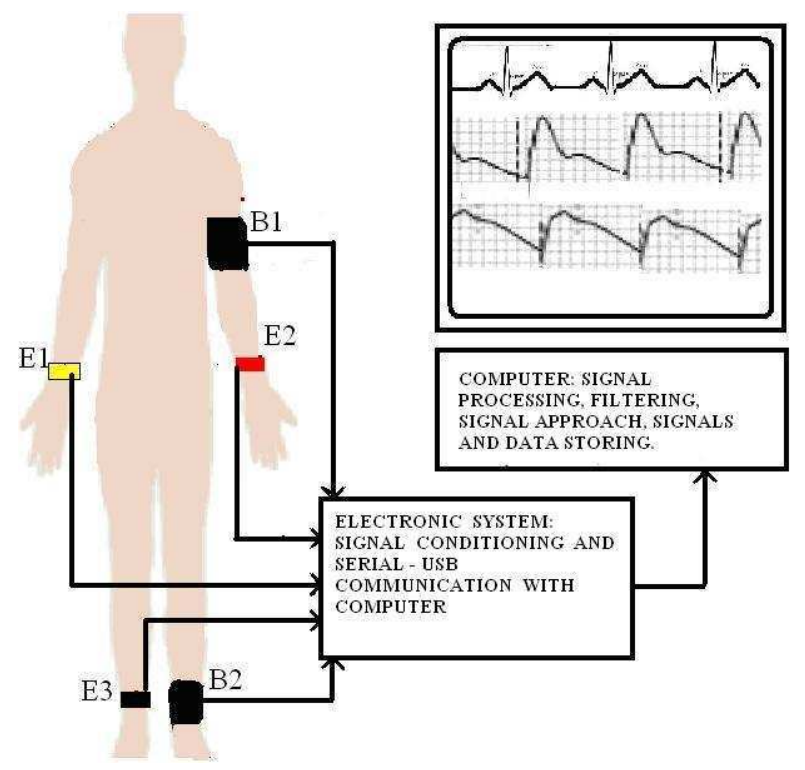

Figure 3. System for measuring baPWV.

The signal conditioning stage is divided in two sections: one for bioelectrical signal (ECG) and another for pulse waves (brachial and ankle). Figure 4 shows the block diagram of ECG signal conditioning. 


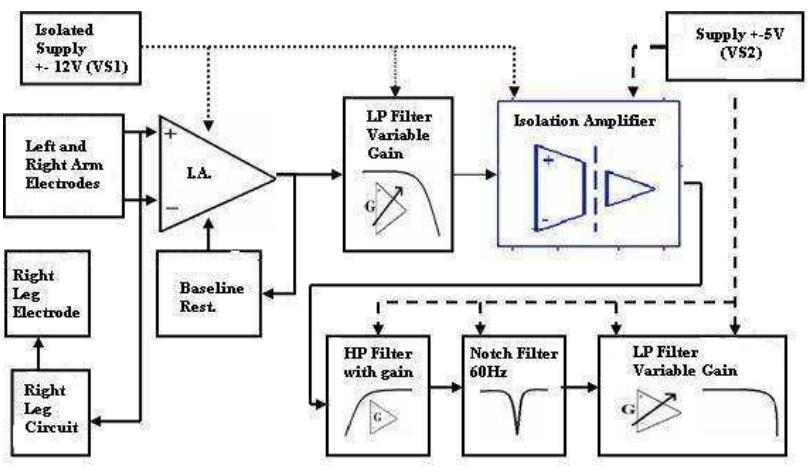

Figure 4. Block diagram of ECG signal conditioning.

The block diagram of pulse wave signal conditioning is shown in figure 5 .

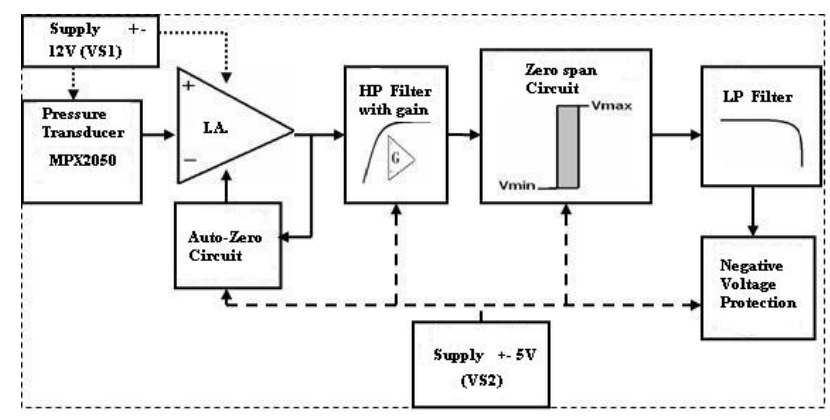

Figure 5. Block diagram of pulse wave signal conditioning.

The acquired signals of volume pulse and ECG were displayed. The acquisition and mathematical processing programs were implemented in object-oriented programming $\mathrm{C}++$.

The reference points of volume pulse wave are minimal and maximal points and maximal time derivative point of waveform, which are shown in figure 6 .

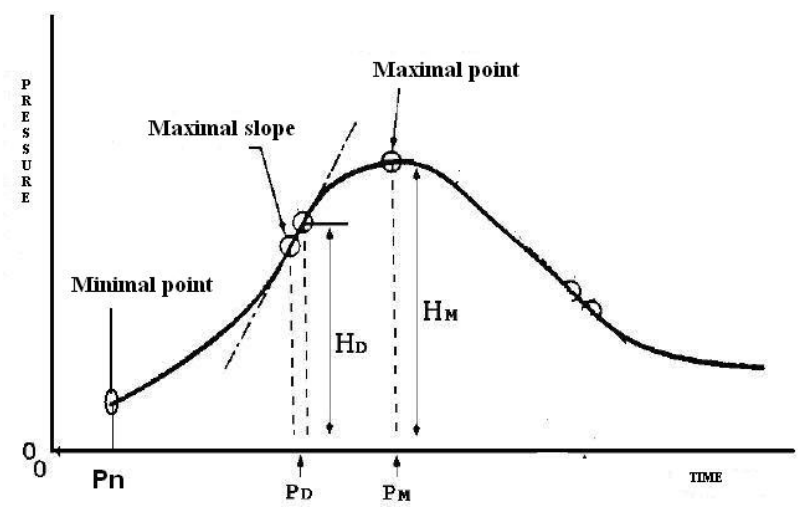

Figure 6. Reference points of volume pulse wave.
The time interval between minimal points as a maximal time derivative points of waveform of the brachial waveform and that of the ankle waveform was defined as pulse transit time $(\Delta t)$. The distance between the brachial and ankle sampling points was calculated based on the height of the subject using next equations [8].

$$
\mathrm{Dba}=\mathrm{da}-\mathrm{db}
$$

where:

$$
\begin{array}{r}
\mathrm{db}=0.2195(\mathrm{x})-2.0734 \\
\mathrm{da}=0.8129(\mathrm{x})+12.328
\end{array}
$$

$\mathrm{x}=$ height of the subject in $\mathrm{cm}$.

With the distance and pulse transit time, PWV is calculated: $\mathrm{PWV}=\mathrm{Dba} / \Delta \mathrm{t}$.

A study was carried out for evaluating the usefulness of these brachial ankle pulse wave velocity measurements with 39 people, 22 healthy volunteers and 17 patients with diagnosed cardiovascular diseases as hypertensión, atherosclerosis or diabetes mellitus. After the volunteers and patients had rested in a supine position at least $5 \mathrm{~min}$, eight brachial ankle pulse wave velocity measurements (four for maximal time derivative points and four for foot-to-foot pulse waves) were measured by subject.

\section{Results}

The analysis of brachial ankle pulse wave velocity is obtained as follows. The acquired signals of brachial and ankle volume pulse waves and ECG are displayed. Figure 7 shows the brachial and ankle volume pulse waves and ECG from a healthy volunteer.

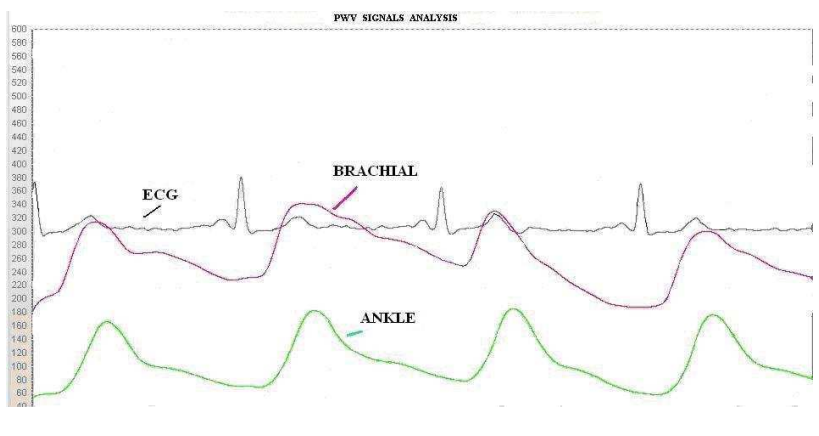

Figure 7. The brachial and ankle volume pulse waves and ECG from a healthy volunteer. 
The reference points of brachial and ankle volume pulse waves and ECG from a healthy volunteer is shown in figure 8 .

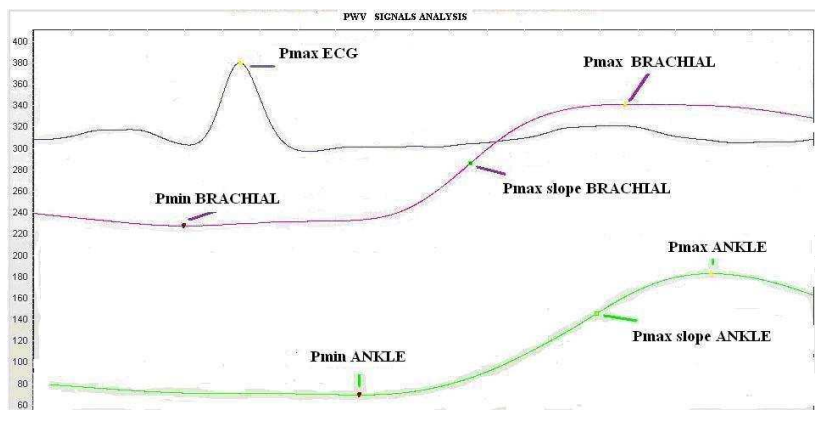

Figure 8. The reference points of brachial and ankle volume pulse waves and ECG from a healthy volunteer.

A t-tested distribution of brachial ankle pulse wave velocity was performed between healthy volunteers and patients using maximal time derivative. It had shown a significant difference $(7.16 \mathrm{vs} 15.08 \mathrm{~m} / \mathrm{s}, \mathrm{p}<0.0001)$. A second $\mathrm{t}$-tested distribution was performed using foot-tofoot pulse waves. It showed a significant difference $(8.08$ vs $19.18 \mathrm{~m} / \mathrm{s}, \mathrm{p}<0.0001)$. However, the variance in patients measurements using foot-to-foot pulse wave was found to be higher than using maximal time derivative (26.25 vs 12.3$)$ and also for healthy volunteers (1.14 vs $0.32)$.

\section{Discussion and conclusions}

The analysis of brachial ankle pulse wave velocity measurements was carried out by an automatic pulse wave velocity device. The calculated parameters have shown significant differences between healthy volunteers and patients using both maximal time derivative and footto-foot pulse waves. However, the variance in patients measurements using foot-to-foot pulse wave was found to be higher than using maximal time derivative and also for healthy volunteers.

In conclusion, automatic brachial ankle pulse wave velocity measurements using maximal time derivative showed to be more accurate than foot-to-foot pulse wave both in patients with cardiovascular disease and in healthy volunteers

\section{References}

[1] Lehmann ED: Clinical value of aortic pulse-wave measurement. Lancet. 1999; 354: 528-529.

[2] Asmar R, Benetos A, Topouchian J, et al: Assessment of arterial distensibility by automatic pulse wave velocity measurement: validation and clinical application studies. Hypertension 1995; 26: 485-490.

[3] Bank AJ, Kaiser DR. Smooth muscle relaxation: effects on arterial compliance, distensibility, elastic modulus, and pulse wave velocity. Hypertension, 1998; 32:356-359.

[4] Blacher J, Guerin AP, Pannier B, Marchais SJ, Safar ME. London GM. Impact of aortic stiffness on survival in end-stage renal disease. Circulation, 1999; 99:2434-2439.

[5] Weber T, Auer J, O'Rourke MF, et al. Arterial stiffness, wave reflections, and the risk of coronary artery disease. Circulation, 2004; 109:184-189.

[6] London G, Blacher J, Pannier B, et al. Arterial wave reflections and survival in end-stage renal failure. Hypertension, 2001; 38:434-438

[7] Yamashina A, Tomiyama H, Takeda K, Tsuda H, Arai T, Hirose K, Koji Y, Hori S, Yamamoto Y. Validity, Reproducibility, and Clinical Significance of Noninvasive Brachial-Ankle Pulse Wave Velocity Measurement. Hypertens. Res. 2002; 25: 359-364.

[8] Yamashina A, Tomiyama H, Arai T, Hirose K, Koji Y, Hirayama Y. Brachial ankle pulse wave velocity as a marker of atherosclerosis vascular damage and cardiovascular risk. Hypertens. Res. 2003; 26: 615-622.

Address for correspondence

Rodolfo Gonzalez

Instituto de Investigación e Innovación en Bioingeniería, UPV

Valencia- Spain.

E mail: rodogon21@yahoo.com 